\title{
Development of a household high-definition video transmission system based on ballpoint-pen technology
}

\section{- A low cost, easily deployed optical connection using a ballpoint-pen type interconnect -} Tetsuya TomA ${ }^{1,2}$ * , Hiroshi TAKIZUKA ${ }^{1,2}$, Toshitaka ToRIKAI ${ }^{3}$, Hitoshi SUzUKI ${ }^{3}$, Tetsuro OGI ${ }^{2}$ and Yasuhiro KoIKE ${ }^{1}$

[Translation from Synthesiology, Vol.7, No.2, p.118-128 (2014)]

\begin{abstract}
Higher quality video formats beyond HDTV are being developed, but these formats require data communication rates greater than 10 Gbps, which are not easily adaptable to household applications. Moreover, household usage requires ease of handling, robustness against poor connectivity, and affordable cost. Conventional silica optical fibers are not suitable for applications that involve high-speed, shortrange communication, because of their fragility, and difficulty in achieving precise connectivity with a low cost connecting method. We proposed a novel expanded-beam interconnect using a graded-index plastic optical fiber (GI POF) with a glass spherical collimator lens uniquely fabricated by low cost ballpoint-pen technologies at both ends. Our power budget evaluation verified its suitability for consumer applications. Successful validation was then conducted by transmitting 4K-3D uncompressed video content.
\end{abstract}

Keywords : Optical communications, collimator lens, optical system design, ballpoint pen, plastic optical fiber

\section{Introduction}

The uncompressed image transmission at home requires quite high bandwidth. Although the current high-definition (HD) television format $(1920 \times 1080$ pixels/60 fps/interlace $)$ provides high quality video for consumers, more advanced video technologies that deliver even higher quality than HDTV are becoming more and more important, because of increased display size of television sets in consumer living rooms. To keep the equal resolution, a television set having twice the size of its display needs double the number of pixels in both the horizontal and vertical directions (four times more pixels in total) compared to that of the HD format and an optional double frame rate for fast moving picture smoothness. The $4 \mathrm{~K}$ format $(3840 \times 2160$ pixels $/ 60 \mathrm{fps} /$ progressive) requires eight times faster data communication than full high definition video format or over $10 \mathrm{Gbps}$ bandwidth. In addition to this, if stereo $3 \mathrm{D}$ viewing also becomes popular, the data size would have to be doubled in order to maintain the same image resolution for each viewing.

To achieve the target transmission speed for such higher definition digital video formats, the existing copper wire communications, such as DVI (Digital Visual Interface) or HDMI (High-Definition Multimedia Interface) are insufficient and optical communications are required. Conventional silica multimode optical fibers (Silica MMFs) can be applied, which are widely used for professional data communication. However, being fragile, they are not appropriate for consumer short-reach applications at home or offices. ${ }^{[1][2]}$ Users may step on them or may wire, bundle, or use them carelessly, that increase risk of fiber breaking. Broken fibers can hurt users and should not be discarded as normal garbage. Moreover, a user has to wipe dust or stains on a fiber end with alcohol or to blow them with clean air spray to ensure its connectivity.

To solve the connecting problem, lens termination of the fiber end improves the ease of use of optical fibers, since it expands and collimates output beam. A ball lens connector is a good practical solution and is called the "expandedbeam connector., ${ }^{[3]}$ Figure 1 compares (a) a conventional butt-coupling connection with (b) a ball lens connection. The expanded-beam output from the ball lens increases the tolerance of both gap distance and axial alignment. The lens also protects the fiber end making it more robust and less sensitive to dust and contaminations and has often been used for heavy duty applications, such as that in vehicles or military circumstances, where ease of connection under rugged conditions is required. ${ }^{[4]}$ In order to attach a ball lens well aligned to the center of the optical fiber core, designing its connector housing needs high preciseness which results in expensive manufacturing cost. Because of this, it has only been applied to special applications. Moreover, its housing becomes bulky and difficult to be made small. To overcome silica fiber fragility against bending, an improved product is available, but its cable is too thick for consumer application with its thick cover material.

Considering these backgrounds, we propose a safe, easy to handle, reliable, and low cost high speed optical communication

1. Keio Photonics Research Institute, Keio University 7-1 Shin-Kawasaki, Saiwai-ku, Kawasaki 212-0032, Japan * E-mail: t.toma@sdm.keio.ac.jp, 2. Graduate School of System Design and Management, Keio University 4-1-1 Hiyoshi, Kohoku-ku, Yokohama 223-8526, Japan, 3. Research and Development Center, Mitsubishi Pencil Co., Ltd. 2-5-12 Irie, Kanagawa-ku, Yokohama 221-8550, Japan

Original manuscript received November 6, 2013, Revisions received November 21, 2013, Accepted November 25, 2013 
system in this study, which enables transmitting uncompressed high resolution video. Specifically, an ultra small optical interconnect having a small ball lens on an end of the plastic optical fiber was developed. A novel connector combining multiple interconnects was then developed and its verification test was run successfully.

\section{Target and Scenario}

Concerning the issues which must be examined in introducing the optical fiber communications system for consumer homes, one should consider not only technical requirements to meet specific transmission speed (bandwidth), but also requirements of safety, ease of handling, simple and reliable connection, and its low cost availability from the consumers' points of view.

Instead of silica optical fibers which are difficult to meet these requirements, plastic optical fibers (POFs) are the most promising candidates as they have better flexibility, physical durability, and can be mass produced at low cost.

POF is strong enough against stamping on or bending tightly so that fiber breakage does not occur, compared with silica optical fibers. A step-index (SI) type POF generally diffused in the market is thought to be appropriate for indoor wiring use, as it does not require special care against axial misalignment or dust and scratches on a fiber end at its connection because of its large core diameter. Considering uncompressed high resolution video transmission of this study, however, since it has narrow transmission band, access speed is insufficient in the zone of several $100 \mathrm{Mbps}$.

On the other hand, perfluorinated graded-index plastic optical fibers (PF GI POF) (Fig. 2) invented by Y. Koike, et al. at Keio University have been commercialized for high speed communication. PF GI POF has better flexibility than silica optical fiber, and the world's fastest GI POF transmits approximately $40 \mathrm{Gbps}$, with POF flexibility advantage. Koike, et al. has proposed "Face-to-Face Communication System" between remote places using a large size high resolution display with the perfluorinated GI POF. ${ }^{[5]}$

Since PF GI POF has similarly wide bandwidth and rather better physical material characteristics than silica optical fibers, Ralph, et al. of Georgia Institute of Technology evaluated it highly. ${ }^{[6]}$ Because it is a plastic material, it is possible to mass produce it with extrusion-molding

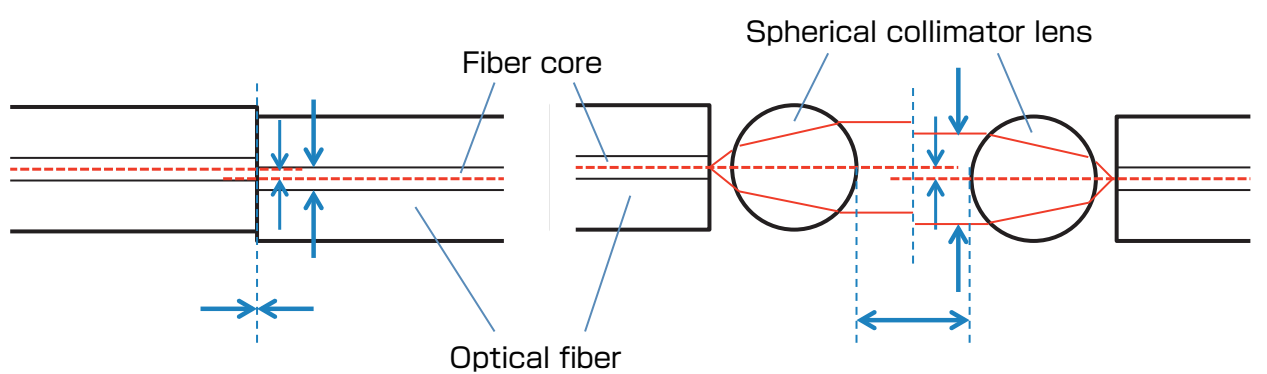

(a) Conventional connection (physical contact + precise alignment) (b) Ball lens connection

(gap tolerance + rough axial alignment)

Fig. 1 Schematic image of ball lens benefits

Conventionally optical fibers should be connected with physical contact and precise axial alignment. A ball lens on the end of optical fiber collimates output light with expanded beam which makes it easier to connect without precise alignment or physical contact.
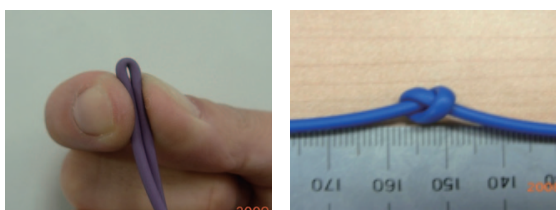

《Strength》

High speed communication

Flexibility (bending resistance)

《Weakness》

$\times$ Softness (weak scratch resistance) $\times$

$\times$ Electrostatic (attracts dust)
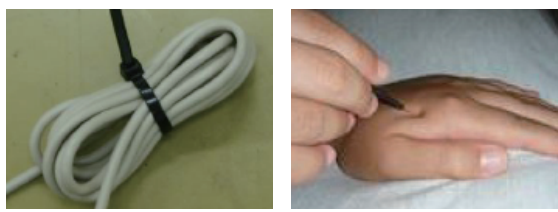

Safe (no dangerous broken piece)

Low cost, continuous mass production

Not suitable for small volume

$\times$ Few adoption results yet

\section{Fig. 2 Strength and weakness of GI POF}

Perfluorinated GI POF (graded index plastic optical fiber) is physically flexible compared with silica optical fibers, and shows excellent bending resistance and is safe against breakage. Thus it is easy for consumers to handle it. However, one has to be careful not to scratch the ends or use it in a dusty environment. 
technology at low cost. However, in order to make highspeed communication possible, the diameter of the fiber core should be designed small and it loses the advantage of ease of connection of POF. The high speed POF requires precise connection with accurate axial alignment and special care for dust and scratches on the fiber ends similar to silica MMF. To keep the ends clean, it should have an end cap for dust prevention and one needs to clean the ends in advance to the connection. As a plastic tends to attract more dust with static electricity and is softer or has lower scratch resistance, such care should be taken even more than silica.

Although it is known that above-mentioned ball lens protects the fiber ends and can improve such failure problems, conventional process of attaching a ball lens is costly and minimizes POF benefits of low-cost short-reach communication business. Since GI POF was invented 20 years ago, even though it has been said to be an ideal solution for home network systems, the application of GI POF has been limited because of these trade-offs. Our study can be an innovative integration technology to breakthrough this fact.

Figure 3 explains the scenario of the development. As already mentioned, an expanded-beam connector having a ball lens on its end has been known to improve connectivity and to prevent dust and scratches on fiber ends. However, if it is used with POF, it is costly; if it is used with silica MMF, it does not meet consumer needs of flexibility. In other words, although the function of the ball lens is excellent, the conventional ball attaching technology cannot be a solution to improve the trade-offs.

Torikai (one of the authors of this paper, worked for Japan Aviation Electronics Industry at that time), on the other hand, was struggling to develop a small optical interconnect for electronics on-board application that can connect the narrow spaces within the equipment as a jumper wire.
Although connection became easy when there was a ball lens, he remembered difficulty in using micro form required for a jumper wire. One day in 2009, he had an opportunity to discuss it with an engineer from Mitsubishi Pencil who produces ballpoint pens in a cross-industrial networking meeting. He realized the similarity of the structural concept of ball attachment on a tip of a thin tube or fiber, although the material of the ball was different; one was glass and the other, metal.

A joint research project between the two companies was initiated right after the meeting. A silica glass optical fiber was first inserted instead of the ink tube of the needle tip ballpoint pen. However, the silica fiber broke easily in the metal sleeves, when it was crimped by a regular punching process to anchor the fiber. In 2010, when the team was almost giving up the development, they met Koike by chance who had just been assigned as a technical advisor to Mitsubishi Pencil. Koike was consulted about the development and suggested to replace silica fiber with GI POF, Koike's invention. He thought that the plastic material similar to the ink tube was flexible and should work.

Furthermore, when using the ballpoint pen technology, attaching a ball lens could realize the low cost solution. It was expected that such a low cost connector with a ball lens could be introduced not only into the electronics field but also into households. As a result of an experiment, GI POF was successfully attached to the needle tip metal sleeve without breakage which made the team re-start the project.

To summarize the scenario, the purpose of the study was to develop basic technology and to verify if it could optically transmit high resolution video for consumer use. We conducted the study with the scenario that the ballpoint pen component technology enables GI POF to be used at low cost with an expanded-beam connector at its end.

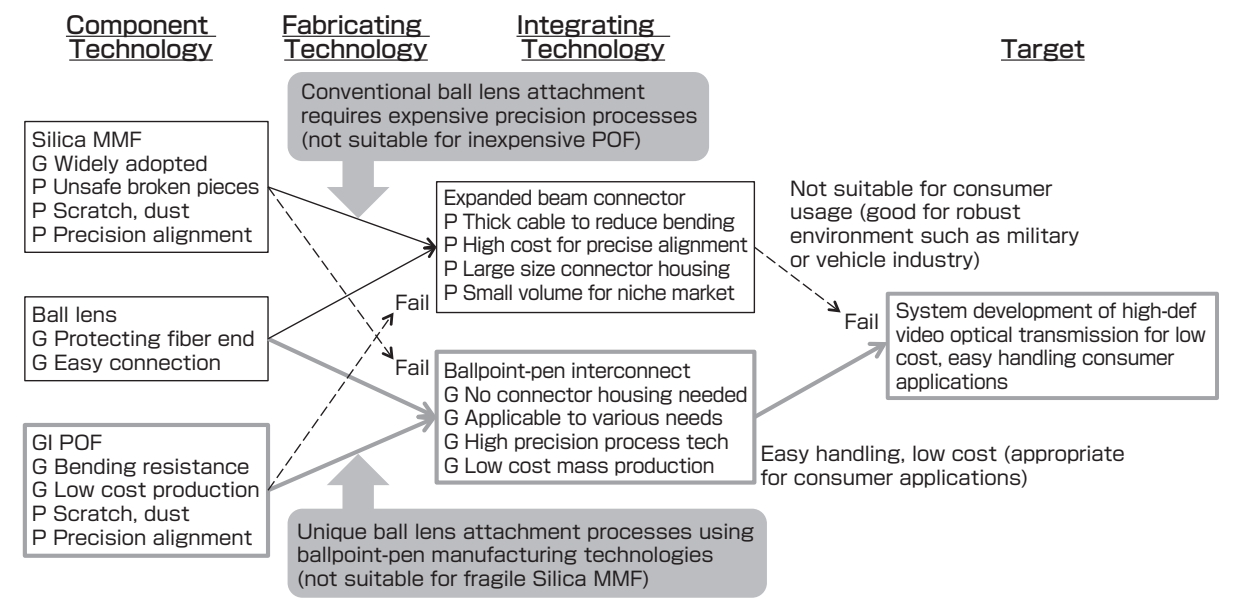

Fig. 3 Scenario of technology development for consumer optical connection

Benefits of ball lens have been well known, but its fabrication requires high, expensive precision processes which means limited usage in robust, special applications. This study enabled low cost, precision ball lens attachment using ballpoint-pen manufacturing technologies. 


\section{Development of ballpoint pen type optical interconnect}

To attach a ball lens on an end tip of the plastic optical fiber, we introduced for the first time in the world a unique manufacturing method-ballpoint pen production processes that considerably reduce process cost. ${ }^{[7]}$ The construction of the unique, novel ball lens attachment concept is shown in Fig. 4.

The tip of the needle-tip type ballpoint pen comprises three components: needle shape metal sleeve (inside diameter 0.5 $\mathrm{mm}$, length $7.8 \mathrm{~mm}$ ), metal ball inserted in the tip (precisely polished to a perfect sphere of $0.55 \mathrm{~mm}$ diameter), and a capillary that helps ink to flow. We kept the metal sleeve asis and replaced the metal ball with a highly transparent glass sphere (material \#BK7, $0.55 \mathrm{~mm}$ diameter, polished precisely to a perfect sphere), then inserted PF GI POF from AGC "FONTEX ${ }^{\circledR}$ " instead of the ink capillary. The metal sleeve is punch processed so that an optical fiber and a glass ball can keep the distance between them precisely. After that, the fiber was fixed by crimping it from the outside of the sleeve so that it could not be removed.

Axial alignment of POF and the ball should be good enough. From this point of view, the ballpoint pen production technology is able to satisfy the need of precise alignment for optical communication, since alignment of these pen components has to be precise in design and manufacturing to minimize writing failure for the ballpoint pen. In addition to this, the precision polishing of the ball to produce ballpoint pens is also the right technology appropriately fit for high quality optical characteristics which require the ball lens to be a perfect sphere with a smooth surface.

Crimping process is also done with the existing technology for ballpoint pen production to prevent inserted optical fiber from removal failure. Flexibility of POF fit quite well with the process technology. The locations and pressure of crimping were adjusted by trial and error within the regular production parameters for ballpoint pens.
These processes were difficult to accomplish for a silica optical fiber. This is the most unique, essential discovery in this study to make use of POF characteristics. In other words, conventional ballpoint pen production technologies, which need precision component alignment, ball polishing and tube crimping, just perfectly fit the needs of the tiny optical interconnection and made it possible to realize it at extremely low cost in manufacturing.

\section{Experimental setup and results}

Experimental procedures and the results to evaluate performance of the newly developed optical interconnect are discussed in this section.

\subsection{Evaluation of connecting loss from misalignment} Optical power meter "Photom 205A" from Graytechnos was used to read optical power transmitted for each condition. We set a pair of optical cables on an apparatus that can precisely control distance and angle of $\mathrm{x}, \mathrm{y}$, and $\mathrm{z}$ axes between the two cable ends. Wavelength of the light source was $850 \mathrm{~nm}$. Dust particles on the ends were carefully removed in advance of the measurement by an air cleaner. The best condition was sought by adjusting the $\mathrm{x}, \mathrm{y}$, and $\mathrm{z}$ axes where the power was maximized and this condition was considered as the base point (the $\mathrm{x}, \mathrm{y}$, and $\mathrm{z}$ locations were zero at this condition). From this point, we measured the optical power in $\mathrm{dBm}$ unit at each different misalignment location. The data was normalized using the maximum power condition.

Connecting loss simulation was run by a ray tracing method using ZEMAX ${ }^{\mathrm{TM}}$. The number of ray tracing trials was 200,000 in order to predict coupling loss distribution by introducing Gaussian distributed misalignment tolerance. In the simulation, GI POF of $0.49 \mathrm{~mm}$ diameter with $55 \mu \mathrm{m}$ core $(\mathrm{n}=1.350, \mathrm{NA}=0.245)$ and a glass ball lens of $0.55 \mathrm{~mm}$ diameter $(n=1.51)$ were used. The distance between the fiber end and the ball was set at the optimized length and was based on our other simulation results (to be reported elsewhere).

Four types of losses can be considered to evaluate total

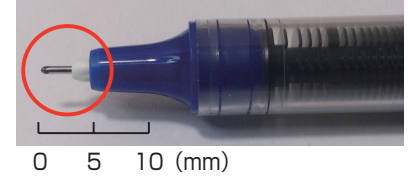

(a) Ballpoint-pen "uni-ball eye"

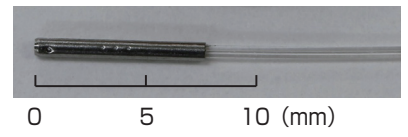

(b) Needle tip with GI POF

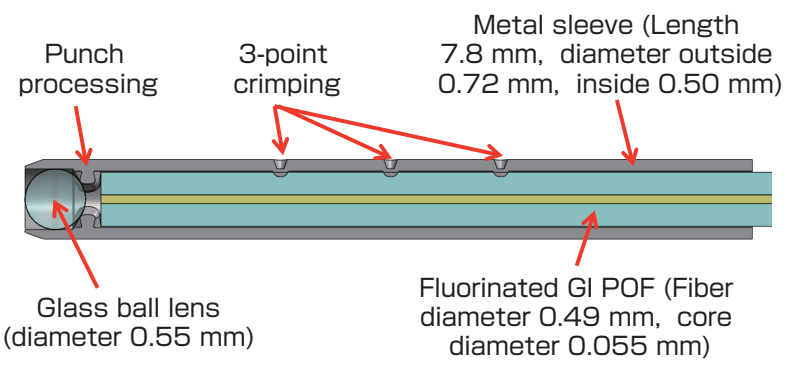

(c) Structure of the tip

Fig. 4 Structure of the ballpoint-pen interconnect

Applying needle-tip ballpoint-pen technologies enables low cost and precise attachment of ball lens. The ball lens is attached not into the connector housing, but directly onto a fiber tip. This invention gives high freedom to connector designing. 
connecting loss: collimation loss, axial misalignment loss, gap tolerance loss, and bending loss. The results of these evaluations are as follows.

\section{(1) Collimation loss}

We made 40 ball-lens-terminated GI POF samples and evaluated the connection loss using one of them as a master. Figure 5 is a histogram of the connection loss data from 39 samples. The average loss was $-1.36 \mathrm{~dB}$. From this result, the coupling loss number we used for power budget estimation (chapter 5) was $2.72 \mathrm{~dB}(1.36 \mathrm{~dB} \times 2$ for both ends of the fiber).

\section{(2) Axial misalignment loss}

Axial misalignment loss was also evaluated, which occurs when a couple of cables were misaligned perpendicular to the axis, comparing with and without ball lens.

Figure 6 shows loss data from axial misalignment. Data was normalized in $\mathrm{dB}$ by setting the coupling loss at the best

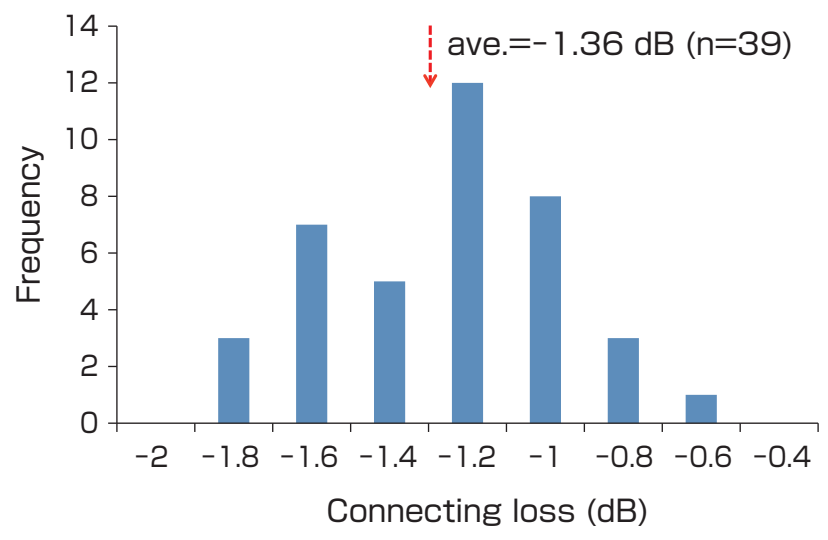

Fig. 5 Connection loss histogram of 39 samples

Shows evaluation results of connecting loss using prototype samples. The average loss is $1.36 \mathrm{~dB}$

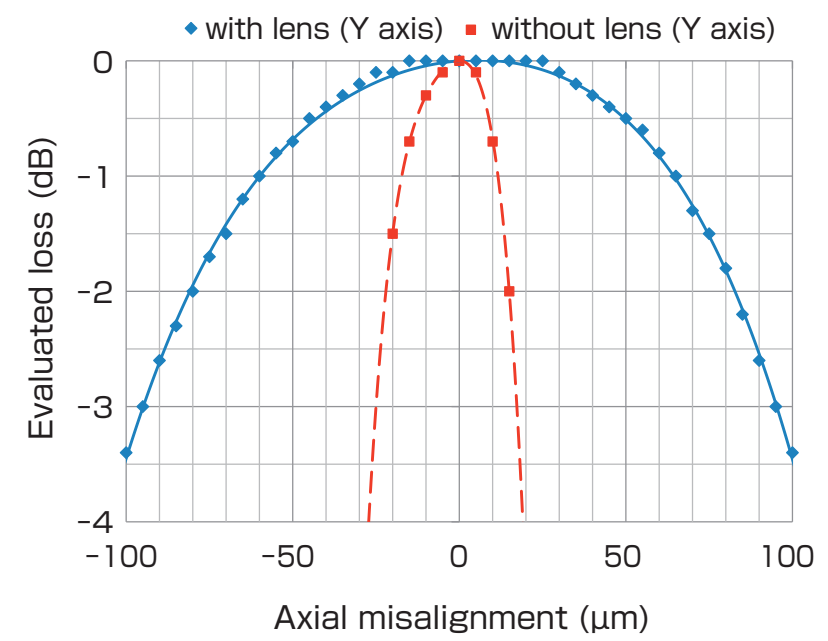

Fig. 6 Connecting loss against axial misalignment

Loss data against axial misalignment perpendicular to the axis. The use of ball lens increases the tolerance margin against axial misalignment. aligned condition to zero. As shown in Fig. 6, the ball lens works quite well to increase the tolerance margin of the axial misalignment.

Without the ball, very precise alignment within $10 \mu \mathrm{m}$ is required to minimize loss below $1 \mathrm{~dB}$, while the ball lens connector can misalign up to $60 \mu \mathrm{m}$, providing six times wider window of tolerance. This feature improves design flexibility for all surrounding parts and devices that will likely decrease manufacturing cost and labor cost for fabrication.

\section{(3) Gap tolerance loss}

Figure 7 shows the gap tolerance loss data. The gap tolerance is typically within $0.05 \mathrm{~mm}$ to keep the power loss below 1 $\mathrm{dB}$. However, once the ball lens is applied to the fiber end, the tolerance becomes much bigger, there is almost no loss up to $0.5 \mathrm{~mm}$, and is still good at $0.8 \mathrm{~mm}$. Collimated beam output from the ball lens provides this significant advantage.

\section{(4) Bending loss}

Bending loss evaluation was conducted comparing GI POF with two commercially available silica multimode optical fibers: "OM2" and "OM3" from Furukawa Electric as representing commercial silica MMFs. As a typical perfluorinated GI POF, FONTEX ${ }^{\circledR}$ from AGC was used. As shown in Fig. 8, bending loss of GI POF was considerably lower than MMFs from Furukawa Electric and this result is promising for home or office network applications. No loss was observed down to $\mathrm{R}=3.5 \mathrm{~mm}$ which simulated tying the cable tightly.

\subsection{Characteristic analysis by light power measurement} To understand characteristics of the optical interconnect we invented, attenuation was evaluated using an optical power meter.

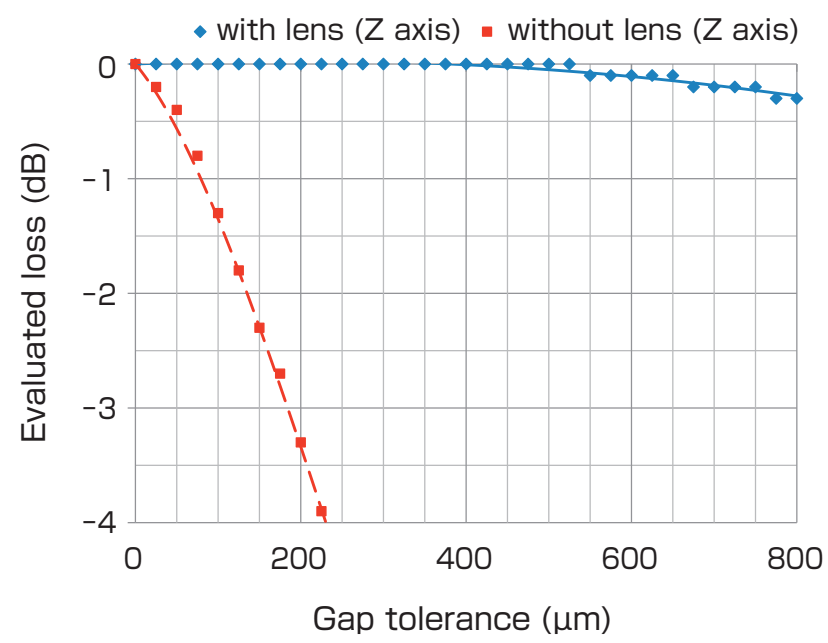

Fig. 7 Coupling loss of gap tolerance

Gap tolerance loss data. With the ball lens, the tolerance becomes much bigger, there is little loss up to $0.8 \mathrm{~mm}$. 
(1) Bit error rate test (BERT) for power penalty

Bit error rate (BER) was measured using BER and EYE/ pulse scope "BERTWave MP2100A" from Anritsu, and optical power was adjusted using optical attenuator "N7766A" from Agilent Technologies. Power penalty (required power increase to make up for a loss in the transmission channel) was calculated as the difference in power value at BER 1E-12 between the sample and back-to-back reference (a standard condition at the lowest loss by directly connecting the transmitter and the receiver).

Power penalty at the low bit error rate (BER) by cable length was also evaluated. Figure 9 (a) shows the loss caused by the cable itself as a function of cable length. It shows almost a linear relationship between the length and the loss. The power penalty loss number for a 100 -m-long cable is $1.12 \mathrm{~dB}$, $50-\mathrm{m}$ is $0.66 \mathrm{~dB}, 20-\mathrm{m}$ is $0.29 \mathrm{~dB}$, and $10-\mathrm{m}$ is $0.11 \mathrm{~dB}$. These data are used for power budget calculation in chapter 5 .

\section{(2) Cable loss evaluation}

PNA-X Network Analyzer "N5242A" and Lightwave Component Analyzer "N4376B" from Agilent Technologies were used for measuring frequency characteristics of the optical fibers, and the DC mode value (zero frequency) was considered as a loss from the cable itself, as a function of its length.

Cable losses as functions of its length were then measured. Figure 9 (b) shows plotted data of cable losses at different cable lengths. From these results, cable loss for a 100-m-long cable is $3.76 \mathrm{~dB}, 50-\mathrm{m}$ is $1.87 \mathrm{~dB}, 20-\mathrm{m}$ is $0.75 \mathrm{~dB}$, and $10-\mathrm{m}$ is $0.38 \mathrm{~dB}$. These values are also used in chapter 5 .

\section{(3) Eye Safety of laser}

Considering light leakage that occurs at cable breakage, eye safety evaluation was conducted using optical power meter "8250A/82311" from ADCMT. As the light source, we used NEC's optical transmitter with $850 \mathrm{~nm}$ light source and their receiver. We evaluated the light power based on the accessible emission limit (AEL) standard for eye safety, considering an emergency case of cable breakage. The AEL value per fiber was calculated as $-1.57 \mathrm{dBm}$, which met the Class 1 eye safety standard (Class 1 is the safest standard value for consumer electronics applications).

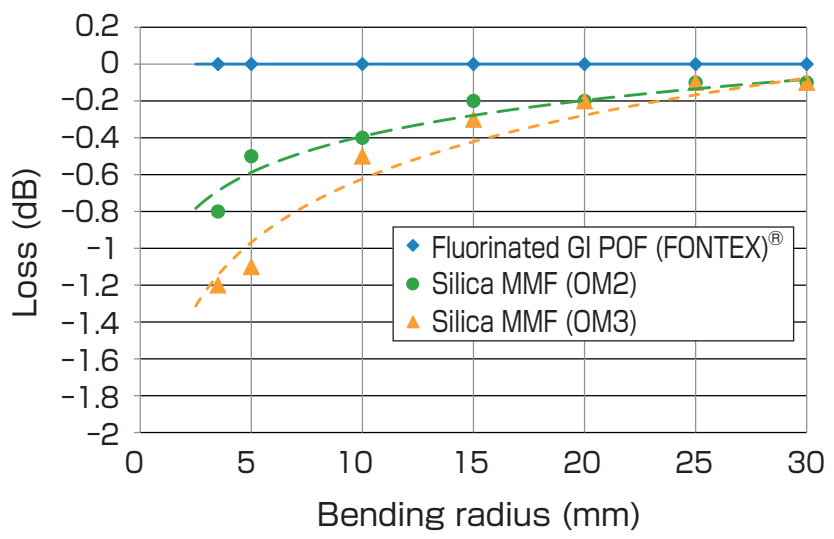

Fig. 8 Bending loss data

Bending loss comparing perfluorinated GI POF with two commercially available silica multimode optical fibers. bending loss of GI POF was considerable lower than MMFs.
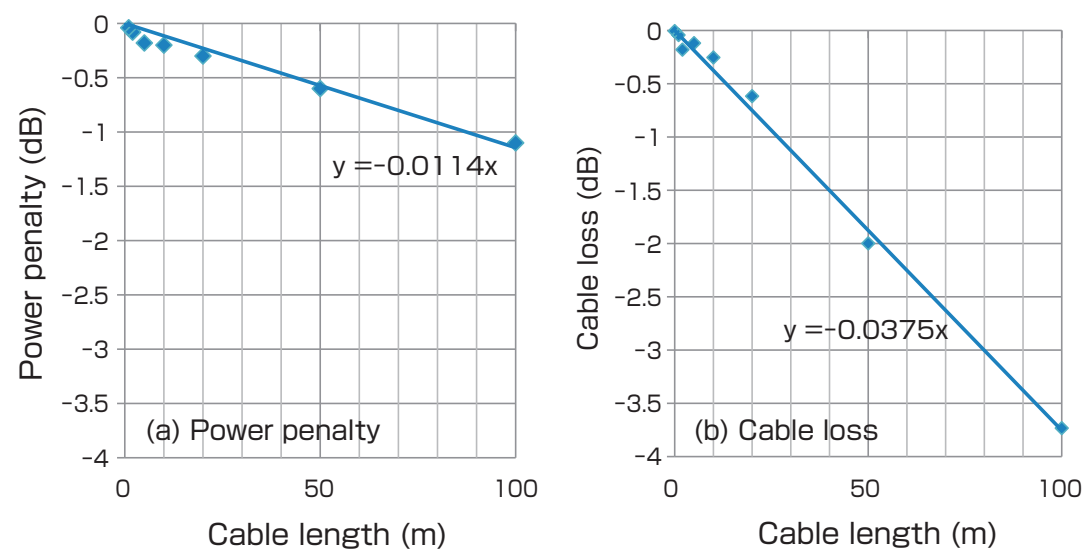

Fig. 9 Power penalty and cable loss by its length

Left (a): Power penalty data and its trend line evaluated by cable length.

Right (b): Cable loss data and its trend line evaluated by cable length. 


\section{Power budget evaluations}

To see if the proposed system is suitable for proper communication, we combined these loss data into the power budget estimation shown in Fig. 10. The graph shows total loss at the cable lengths of $10 \mathrm{~m}, 20 \mathrm{~m}$ and $50 \mathrm{~m}$ from four possible loss factors: collimation loss, alignment loss, power penalty, and cable loss. The target power budget value $7.43 \mathrm{~dB}$ was taken from Stressed Receiver Sensitivity (SRS) of IEEE802.3ae, which is $-9 \mathrm{dBm}$, minus Launch Power considering eye safety, which is $-1.57 \mathrm{dBm}(9 \mathrm{~dB}-1.57 \mathrm{~dB}$ $=7.43 \mathrm{~dB}$ ).

Collimation loss $(2.72 \mathrm{~dB})$ was calculated by doubling the average of individual collimation loss shown in Fig. 5 $(1.36 \mathrm{~dB})$, as the loss occurs at each end of the fiber cable. Alignment loss $(2.04 \mathrm{~dB})$ was calculated from the total loss of the assembled connector having 8-channel GI POF fibers to be discussed in chapter 6 , minus connecting loss of each channel from Fig. $5(4.76 \mathrm{~dB}-2.72 \mathrm{~dB}=2.04 \mathrm{~dB})$. In other words, the total of collimation loss and alignment loss is the insertion loss of the connector. The cable loss data were calculated by using the coefficient of the trend line obtained in Fig. 9 (b). The power penalty data were similarly calculated using Fig. 9 (a).

The combined power budget for the 10-m-long GI POF was $5.25 \mathrm{~dB}$, including insertion loss of $4.76 \mathrm{~dB}$, which is independent of cable length, and power penalty $(0.11 \mathrm{~dB}$ at 10 -m-length) plus cable loss ( $0.38 \mathrm{~dB}$ at 10 -m-length), which are dependant on cable length. There was enough margin of $2.18 \mathrm{~dB}$ against the target budget $(7.43 \mathrm{~dB})$. The length of 10 meters is enough for in-room wiring of most consumer electronics. It is also the length between the devices of our system test prototype described in section 6.2.

Other losses coming from axial misalignment, gap tolerance,

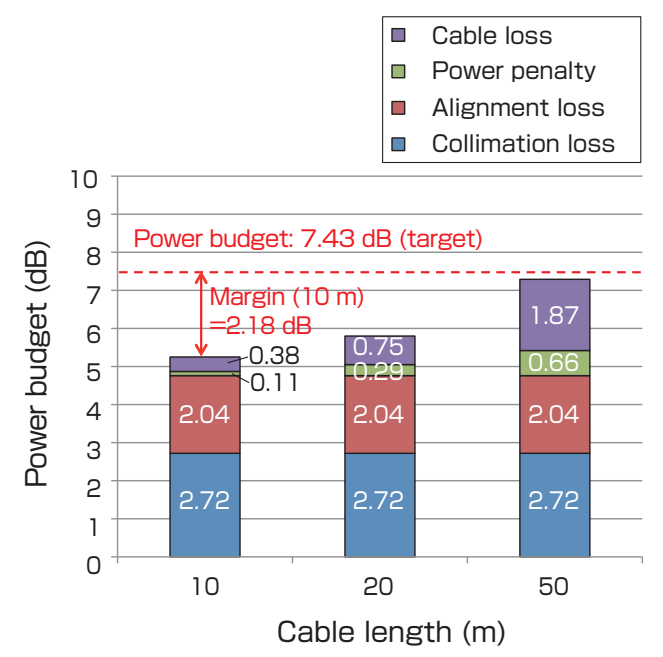

Fig. 10 Accumulated loss and power budget

Evaluated power budget margin by accumulating measured losses. The margin of 10-m-long cable is over $2 \mathrm{~dB}$. 50-m-long cable still has slight margin which is promising for future home network applications. and bending shown in Figs. 6-8 were not included in Fig. 10 , assuming the cables were connected at the optimal conditions. In other words, if there is enough margin against the budget, these alignments do not have to be strictly precise.

As we discussed in chapter 4, ball lens attachment proposed in this study improved optical connectivity to that with little optical loss even if a consumer connects the cable roughly. This fact enables designing optical connection easily with enough margin of power budget. It also influences tolerance of other components and quality control processes which results in possible cost reduction. In contrast, with the conventional butt-coupling optical connection, it is quite difficult to achieve low optical loss under power budget without very precise connection, and it has a high risk of disconnection which makes it not recommendable for consumer home use. Improving connectivity by increasing preciseness is costly and makes it difficult to be handled by a consumer. Therefore, it is thought that this suggested system has enough performance and saves cost to tolerate the home optical network use and it has high flexibility for designing home wiring applications.

As the data in Fig. 10 show, power budget becomes tighter when the cable becomes longer. When a 20 -m-long cable is considered, doubling the length makes it possible to connect electronics between rooms at home. The $20 \mathrm{~m}$ GI POF power budget was $5.80 \mathrm{~dB}$; it still has more than $1 \mathrm{~dB}$ margin to the target $(1.63 \mathrm{~dB})$.

Our target is to apply the new cable to home network. Up to a length of $50 \mathrm{~m}$, the novel GI POF interconnects can be used under target budget. Since the margin of the length is tight $(0.14 \mathrm{~dB})$, it has a high risk of implementation. However, as we discussed, our proposed ball lens system has very low connecting loss. Prospect for a home network using the cables up to 50-m-length is optimistic. These results were encouraging when considering the new interconnect to be used in the home network market. To extend the cable for longer applications in the future, we need to think about reducing alignment loss of $2.04 \mathrm{~dB}$ that comes from multichannel connector variables.

\section{Verification of uncompressed video data transmission}

\subsection{Optical / HDMI-like hybrid interface}

In this chapter, results of verification experiments are reported of transmitting high-definition video through a prototype of optical cable connector using the ballpoint-pen technology.

The use of optical interconnections is still limited in consumer electronics applications. Considering a practical use, it is 
not easy to replace all of video transmission connectors with optical ones. As a transition period, it might be better to develop a connector which can be used for both electric and optical input/output.

An active optical cable (AOC) has also been developed in the market which receives signals electrically, transmits them optically by E/O (electrical to optical) conversion, and delivers them electrically by $\mathrm{O} / \mathrm{E}$ (optical to electrical) conversion. However, we instead developed a prototype of an advanced optical/electrical hybrid connector, to maintain backward compatibility with existing electrical lines for lower speed control signals and existing power supply.

Our newly developed hybrid connector that combines HDMI-like interface with our new optical interface is shown in Fig. 11. As an existing electrical line, we adopted an extension of the HDMI format, because of its reliability for consumer electronics having secured copyright protection. Compatibility with the current HDMI interface is one of the most important requirements to design the interface, which

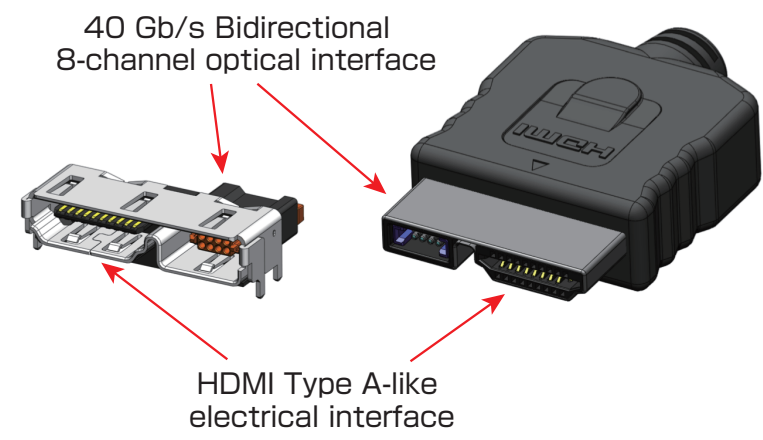

Fig. 11 Prototype of optical/electrical hybrid connector Having a conventional HDMI-like interface, the connector also has 8-channel ballpoint pen interconnects for high speed optical communication to transmit uncompressed ultra high definition video. enables consumers and device makers to transit the system smoothly. To achieve $40 \mathrm{Gbps}$, we chose a four-channel system having four optical cables of $10 \mathrm{Gbps}$ quality for each direction (eight channels for both directions), which enables us to develop a product cheaper and faster using existing technologies, compared with using one cable of $40 \mathrm{Gbps}$ which would be the ideal solution for the future.

The prototype is a pair of receptacle and plug as shown in Figure 11. The left side of the receptacle or the right side of the plug is an HDMI-like electric connector for normal HD content transmission. The other side is the newly developed optical connection for $40 \mathrm{Gbps}$ bidirectional transmission having eight channel ball lens connectors.

\subsection{System testing by transmitting 4K3D uncompressed video} Using the prototype developed in this study, we successfully verified the system performance at Keio University by communicating between two remote locations interactively using 4K-3D 60i uncompressed, synchronized video (3840 $\times$ 2160 pixels/60 fps/interlace, 3D dual stream) for one direction and Full HD 60p uncompressed video $(1920 \times 1080$ pixels/60 fps/progressive) for the other direction. No interruption or pauses occurred during the test.

The 4K-3D video used is an approximately 10 -minute duration film which was shot and edited by Keio University in collaboration with NHK Media Technology Inc. in 2010 using two $4 \mathrm{~K}$ cameras from Red.com Inc. with a 3D rig. The video was stored and played by UDR-20S from Keisoku Giken Co. Ltd.

Figure 12 shows the system structure describing devices and their connections used in the verification test. The experimental run was conducted in May 2012 at the Concurrent Design Facility (CDF) at Hiyoshi Campus, Keio

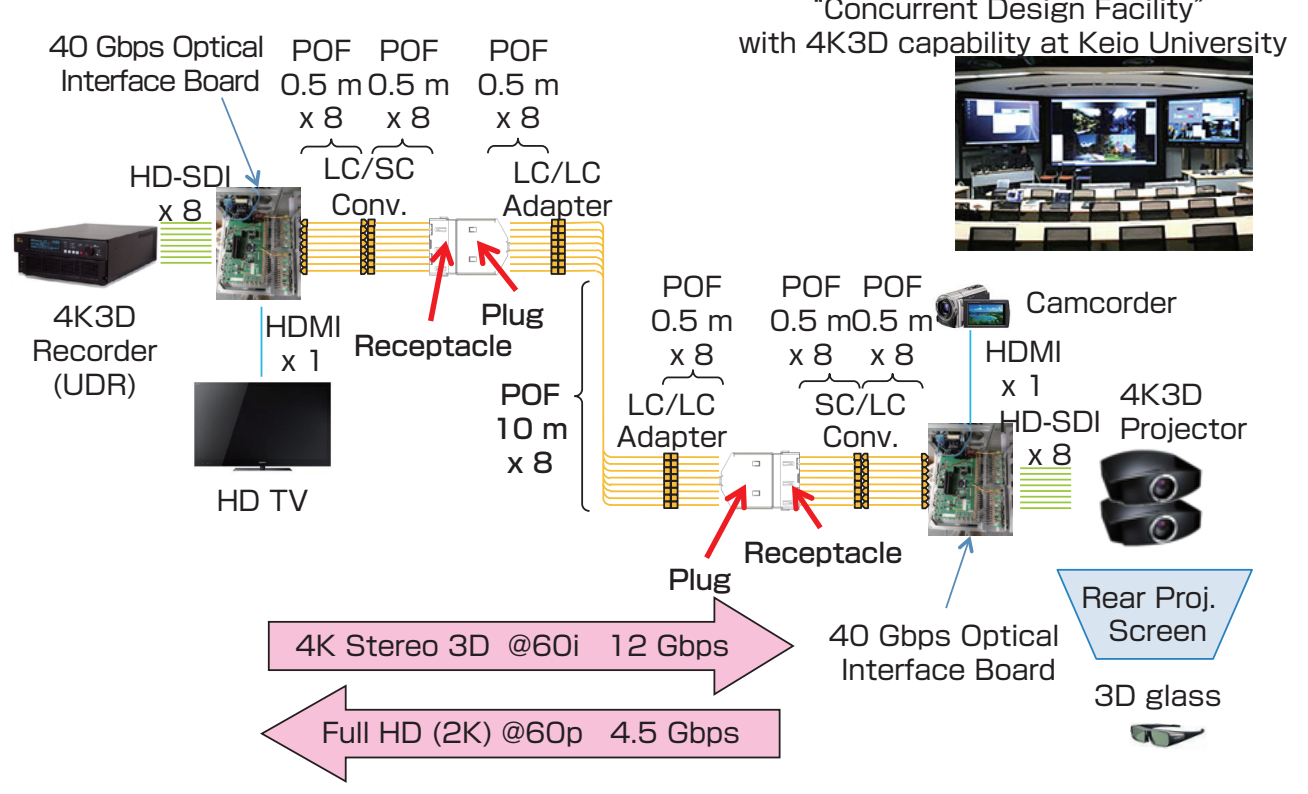

Fig. 12 System structure of 4K3D video transmission Using the prototype of hybrid connector with ballpoint pen optical interconnects, successful verification test was conducted by communicating uncompressed $4 \mathrm{~K}$ $3 \mathrm{D}$ video in one direction and full HD video in the other direction. 
University and proved that the uncompressed $4 \mathrm{~K}-3 \mathrm{D}$ video transmission was promising using the connector.

\section{Conclusions}

In this study, we have proposed an uncompressed highdefinition video transmission system for future consumer electronics using ballpoint pen manufacturing technologies, which enables safe, easy and reliable optical connections through high speed optical communication. Specifically, we developed an extremely small interconnect using perfluorinated GI POF with a glass spherical collimator lens on its each end, and an optical/electrical hybrid connector which includes eight optical interconnects in one cable. Successful verification of the hybrid connector was then conducted by transmitting high definition uncompressed video contents.

The feature of our newly proposed ballpoint pen type optical interconnect is its small size connection without a bulky housing attaching a ball lens directly on the fiber tip, compared with conventional bulky, costly housing design combining a ball lens with an optical fiber precisely. The novel optical interconnect can be used as a jumper wire without a connector or a connecting adaptor. By bundling multiple fibers into one connector, it can conveniently be used for ultra high speed communication for consumer applications.

Moreover, because the ballpoint pen production processes are applied, the ball lens attachment can be mass produced at extremely low cost and is highly practical and almost a complete method to achieve high precision alignment. Not just showing its optical performances, ease of connectivity was practically proved in this paper as well. Optical loss was minimized, even when the axial alignment and gap tolerance were not perfect.

The power budget evaluation results suggested further improvements are required for longer than 50-m length application, such as in home optical network. However, the results showed promisingly that short reach applications between devices up to 10 -meter length are highly practical.

\section{Acknowledgements}

This research has been supported by the Japan Society for the Promotion of Science (JSPS) through its "Funding Program for World-Leading Innovative R\&D on Science and Technology (FIRST Program)." We would like to thank Yuji Watanabe, Asahi Glass Company and Akihito Mitsui, Mitsubishi Pencil for helping us by providing samples and evaluating them. Also many thanks to Prof. Taketoshi Hibiya, Keio University for his guidance and valuable suggestions to this research work.

\section{References}

[1] P. Polishuk: Plastic optical fibers branch out, IEEE Commun. Mag., 44 (9), 140-148 (2006).

[2] I. Mollers, D. Jager, R. Gaudino, A. Nocivelli, H. Kragl, O. Ziemann, N. Weber, T. Koonen, C. Lezzi, A. Bluschke and S. Randel: Plastic optical fiber technology for reliable home networking: overview and results of the EU project POFALL, IEEE Commun. Mag., 47 (8), 58-68 (2009).

[3] J. C. Baker and D. N. Payne: Expanded-beam connector design study, Applied Optics, 20, 2861-2867 (1981).

[4] R. J. Pimpinella: A new type of fiber optic connector designed for military optics backplanes, IEEE Trans. Comp. Hybrids Manufact. Technol., 15, 992-997 (1992).

[5] Y. Koike and T. Ishigure: High-bandwidth plastic optical fiber for fiber to the display, J. Lightwave Technol., 24 (12), 4541-4553 (2006).

[6] P. Decker, A. Polley, J. Kim and S. Ralph: Statistical study of graded-index perfluorinated plastic optical fiber, $J$. Lightwave Technol., 29 (3), 305-315 (2011).

[7] T. Torikai, T. Yamauchi, S. Mine, N. Moriya, A. Mitsui, H. Suzuki, Y. Watanabe, T. Toma and Y. Koike: Optical I/O connectors employing ball-point pen type optical collimator lenses suitable for plastic optical fiber communications, Proceedings of 21th International Conference on Plastic Optical Fibers (POF2012), (Atlanta, USA, 2012).

\section{Authors}

\section{Tetsuya TomA}

After obtaining a master's degree in Applied Chemistry from Keio University in 1988, joined Sumitomo 3M. Moved to 3M Company in USA in 2001 and worked as an advanced product development specialist until 2007. After serving for 20 years in $3 \mathrm{M}$, he joined Keio University in 2008 and is researching communication systems. Received Ph.D. in System Design and Management in 2014. Lead a sub-team to develop optical home network system on the FIRST Program of the Cabinet Office of Japan since 2010. Contributed as a team leader of this project and wrote this paper.

\section{Hiroshi TAKIZUKa}

Completed M.S. degree in Electrical Engineering at Keio University and joined Sony Corporation in 1978. Developed technologies for home network and introduced variety of products into the market; such as OP i.Link, xvYCC color and HDMI standard,s for example. Became a researcher of Keio Photonics

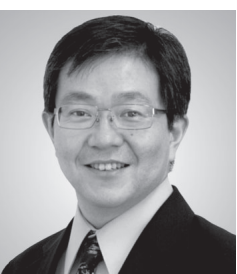
Research Institute in 2013, after finishing work at Sony. For this study, designed the optical interconnect system and optimized it by evaluating its characteristcs. 


\section{Toshitaka TORIKAI}

Received B.S. in Electronic Engineering from Tottori University and joined NEC in 1977. Developed optical communication devices in the laboratories. Moved to Japan Aviation Electronics in 2004 and engaged in optical interconnection development until 2013. Became an independent technical consultant and is advising Mitsubishi Pencil. Co-invented

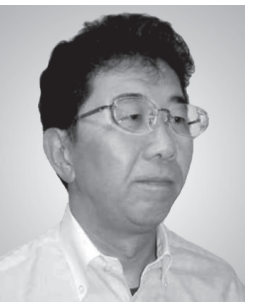
the basic concept of the ball-pen lens optical interconnect and developed the optical/DMI-like hybrid connector for this study.

\section{Hitoshi SUZUKI}

Completed B.S. course in Mechanical Engineering at Saitama University and joined Mitsubishi Pencil in 1983. Moved to a subsidiary of the company in USA in 1993 until becoming Leader of Sharp Pencil Group at its Yokohama R\&D Center in 2002. Promoted to the Head of the R\&D Center in 2005 and became a Director of Mitsubishi Pencil in charge of the R\&D Center. For this study, lead and managed the ballpen collimator lens development team at Mitsubishi Pencil.

\section{Tetsuro OGI}

Obtained a master's degree in Mechanical Engineering from the University of Tokyo, and joined Mitsubishi Research Institute in 1986. Earned a Ph.D. in Mechanical Engineering from the University of Tokyo in 1994 and was named associate professor in 1996. In 2004, he moved to the Graduate School of Systems and Information Engineering, University of

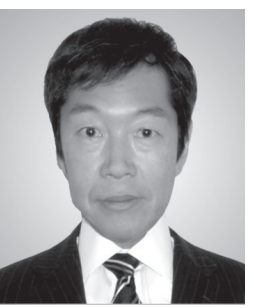
Tsukuba as an associate professor. In 2008, he moved to the Graduate School of System Design and Management, Keio University. His research field includes systems engineering, virtual reality, human interface, etc. Served as an advisor of this project from the network technology standpoint.

\section{Yasuhiro KoIKe}

Earned his B.S. at Keio University in 1977, M.S., in 1979 and Ph.D. in 1982 in applied chemistry at the Graduate School of Engineering of Keio University. Has been Professor at Keio University since 1997. Specializes in "photonics polymer" such as graded-index polymer optical fiber (GI POF), highly scattered optical transmission (HSOT) polymer,

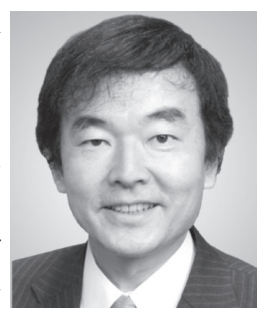
zero birefringence polymer, etc. He has been pursuing an $\mathrm{R} \& \mathrm{D}$ project of Face-to-Face Communication system on the FIRST Program of the Cabinet Office of Japan since 2010. He is a recipient of International Engineering and Technology Award of the Society of Plastics Engineers, the Fujiwara Award, and Medal with Purple Ribbon from the government, etc. Councilor, Keio University. The material and the basic concept of this study were his original inventions.

\section{Discussions with Reviewers}

\section{Overall evaluation \\ Comment (Naoto Kobayashi, Center for Research Strategy, Waseda University)}

This paper shows successful development of an optical interconnect that combines a ball lens and a plastic optical fiber by using ballpoint-pen production technology, and the actual verification test results using the optical interconnect to realize a high-definition video optical transmission system for home use which has to meet extremely severe demands such as highperformance, simplicity and easiness of use, inexpensiveness, safety and security. The paper is valuable not just because it describes the unique component technology, but also because it shows demonstration results using an actual system in which the unique technology is integrated. Thus, the paper is appropriate for Synthesiology.

Comment (Hiroshi Ishikawa, AIST)

I highly evaluate the low-cost optical coupling using the ballpoint-pen technology as an important development for the wide application of POF. In addition, it is an interesting fact that an opportunity for cross-industrial networking led to this technology development.

\section{Scenario \\ Comment (Motoyuki Akamatsu, AIST)}

The key point of this technology is realizing the combination of ballpoint-pen type interconnects with fluorinated GI POF, and the scenario is written in chapter 2. Merits of GI POF are written in several paragraphs in the first half of chapter 2, but it says in the sixth paragraph that "the ball lens attachment was impossible using POF." The ball connector is mentioned from the seventh paragraph, and in the ninth paragraph, it says that you "came across GI POF by chance." It seems the paper is saying that the research was based on the thought that POF exists in the first half, but, on the other hand, in the second half, it says that POF was found after trials to solve fragility problems of silica optical fiber when it was put into the metal sleeve of the ballpoint-pen tip to make a ball lens connector cheaply. There seem to be two different scenarios of opposite directions which confuse readers: one goes from POF to ball connector and the other goes from ball connector to POF. You have to write clearly which scenario was close to the real fact or whether both ran concurrently. Answer (Tetsuya Toma)

There were two groups who had developed an optical connection independently. One day they met by chance and noticed each other's common points which then led to this joint study. I rewrote the description on this part to make it clearer.

\section{Wave length and bandwidth of plastic fiber Comment (Hiroshi Ishikawa)}

You should write the wavelength of the light source somewhere in the paper, though I believe that POF is used in the $0.8 \mu \mathrm{m}$ area. In addition, you should better explain briefly the reason why a bandwidth of SI POF is limited differently from the one of GI POF, as I think that the majority of readers do not know the causing factor. I am familiar with the dispersion of silica fiber, but I not familiar with that of POF.

\section{Answer (Tetsuya Toma)}

Because absorption of carbon-hydrogen bonding exists in the infrared region for regular acrylic polymers, one should use the visible light region for communication. However, if you use the perfluorinated polymer instead, absorption of the infrared region falls. We used $850 \mathrm{~nm}$ this time (described in chapter 4), but the perfluorinated polymer actually works similarly to silica fiber 
using wavelengths between 700 and $1300 \mathrm{~nm}$, or rather it allows faster transmission speed because of its lower material dispersion. In addition, a pulse spreads broadly for SI type fiber, because a light ray that travels through the center of the core and another that travels with many total reflections at an interface of the core and clad have different path lengths within the fiber. On the other hand with the GI type fiber, all the light rays travel without a reflection in the core where refractive index distribution profile has been designed properly, and propagate at the same speed to the end of the fiber by drawing a sine curve. This results in the GI fiber being able to transmit more pulses per second at a wider bandwidth than SI fiber.

\section{Ballpoint pen technology}

Comment (Naoto Kobayashi)

I think that the important key technology in this study was the combination of lens system adoption using ballpointpen production technology with a plastic optical fiber. The experimental results were excellent, but I want to know if there were any particular difficult points in achieving the technology.

\section{Answer (Tetsuya Toma)}

As I wrote in the paper, crimping a plastic optical fiber was physically easy to fix to the metal sleeve without breakage, but it was not so easy to optimize the crimping condition in fixing without loss.

\section{Cross-industrial networking}

\section{Comment (Naoto Kobayashi)}

About developing the optical interconnect using the ballpoint-pen production technology which is the critical point of this study, there was an indication that the cross-industrial networking was important to make this invention happen. Please tell us if you have any thoughts on an active, conscious method to bring about innovation through such a cross-industrial cooperation in the future.

Answer (Tetsuya Toma)

At an opportunity of cross-industrial networking, I believe that it is important to find a common point by conceptually regarding each other's technology from a higher level of abstraction. I think that a new idea is born by abstracting system elements from both physical and functional viewpoints.

\section{Competition with wireless technology} Comment (Naoto Kobayashi)

Considering Tokyo Olympic and Paralympic Games in 2020, the need of the high-definition video transmission for home use will be increasing in the future. However, on the other hand, wireless technology such as LTE has been and will also be rapidly progressing. As a result, it can be said that optical fiber communication only needs to transmit data to the home except in some special cases such as ultra high-definition video, and it is reasonable to use wireless communication in the home. Please tell us about the competition and segregation between optical fiber communication and wireless communication for home use in the future.

\section{Answer (Tetsuya Toma)}

Because transmission speed of wireless is slower than wired and the quality of communication of wireless varies by environment conditions, the transmission of more than $10 \mathrm{Gbps}$ needed to transmit uncompressed SHV of $4 \mathrm{~K}$ and $8 \mathrm{~K}$ is difficult. In addition, the higher the frequency increases (tens of $\mathrm{GHz}$ or more), wireless becomes easily affected by the environment conditions; the transmission may fail when a person walks by, or it may not be easy to pass beyond a wall. I don't think that there is a merit of wireless for the high-speed transmission over $10 \mathrm{Gbps}$. In addition, additional care has to be taken for security management of wireless compared with wired communication. 